International Journal of Modern Physics: Conference Series

(C) World Scientific Publishing Company

\title{
Bulk and edge quasihole tunneling amplitudes in the Laughlin state
}

\author{
Zi-Xiang $\mathrm{Hu}$ \\ Department of Electrical Engineering, Princeton University, Princeton, New Jersey 08544, USA \\ Ki Hoon Lee \\ Asia Pacific Center for Theoretical Physics, Pohang and Department of Physics, Pohang \\ University of Science and Technology, Pohang, Gyeongbuk 790-784, Korea \\ Xin Wan \\ Zhejiang Institute of Modern Physics, Zhejiang University, Hangzhou 310027, P. R. China \\ Received Day Month Year \\ Revised Day Month Year
}

\begin{abstract}
The tunneling between the Laughlin state and its quasihole excitations are studied by using the Jack polynomial. We find a universal analytical formula for the tunneling amplitude, which can describe both bulk and edge quasihole excitations. The asymptotic behavior of the tunneling amplitude reveals the difference and the crossover between bulk and edge states. The effects of the realistic coulomb interaction with a background-charge confinement potential and disorder are also discussed. The stability of the tunneling amplitude manifests the topological nature of fractional quantum Hall liquids.
\end{abstract}

Keywords: Laughlin state, quasiparticle tunneling, Jack polynomial, scaling, disorder

PACS numbers: 73.43.Cd, 73.43.Jn

\section{Introduction}

Fractional quantum Hall (FQH) liquids are examples of experimentally realizable phases that support topological objects. Quasiparticle excitations in the FQH liquids have fractional charge and obeys fractional statistics!12|3 Some FQH liquids may support more exotic quasiparticle excitations with non-Abelian statistics, which have potential applications in topological quantum computation. $\frac{4}{4}$ The measurement of the transport properties of the quasiparticles propagating along the edge of FQH liquids is crucial for the identification of the topological nature of the systems. As standard practice in noise and interference experiments quantum point contacts are introduced to allow quasiparticles propagating on one edge to tunnel to another. This motivated the authors and their collaborators to study the quasiparticle tunneling amplitudes in FQH liquids in the disk geometry $[5]$ We found that the tunneling amplitudes exhibit interesting scaling behavior, whose exponent is related to the conformal dimension of the tunneling quasiparticles. 
In the disk geometry with an open boundary edge excitations arise from the bosonic density deformation of the FQH liquids and, in the case of the MooreRead state, from an extra branch of Majorana fermion mode. The edge excitations are closely related to the bulk quasihole excitations. For example, a charge $|e| / m$ quasihole at $\xi$ in a $\nu=1 / m$ Laughlin droplet $\Psi_{L}=\prod_{i<j}\left(z_{i}-z_{j}\right)^{m} \exp \left(-\sum_{i}\left|z_{i}\right|^{2} / 4\right)$ of $N$ electrons can be described by the wavefunction

$$
\Psi_{q h}=\left[\prod_{i}\left(z_{i}-\xi\right)\right] \Psi_{L},
$$

where $z_{j}=x_{j}+i y_{j}$ is the complex coordinate for the $j$ th electron. The quasihole excitation can be expanded into a sum of edge excitations, whose amplitudes depend on the location of the quasihole $\xi$

$$
\Psi_{q h}=\left[\sum_{n}(-\xi)^{N-n} s_{n}\right] \Psi_{L},
$$

where $s_{n}=\mathcal{S}_{N}\left(\prod_{i}^{n} z_{i}\right)$ is a symmetric polynomial of degree $n$ and $\mathcal{S}_{N}$ denotes the total symmetrization among the $N$ coordinates. The first few examples are $s_{0}=1, s_{1}=\sum_{i} z_{i}, s_{2}=\sum_{i<j} z_{i} z_{j}$, etc. In a Laughlin state the gapless edge mode $s_{n} \Psi_{L}$ spans the Hilbert space of low-energy edge excitations. In fact, there is no strict distinction between a quasihole and an edge excitation with a large angular momentum $\Delta M$. The conventional understanding is that an edge excitation has $\Delta M=O(1)$, while a quasihole excitation $\Delta M=O(N)$. The correspondence of the bulk and edge excitations suggests that the bulk and edge quasihole tunneling amplitudes may have a parallel correspondence and, therefore, a smooth crossover.

In this paper we confirm that the quasihole tunneling amplitude between the Laughlin state and its bulk and edge excitations $\left(\left\langle\Psi_{q h}|\mathcal{T}| \Psi_{L}\right\rangle\right.$ and $\left\langle s_{n} \Psi_{L}|\mathcal{T}| \Psi_{L}\right\rangle$, where $\mathcal{T}$ is the tunneling operator to be defined below), can be described by a unified picture. In particular, we conjecture a universal formula in the limit of a small interedge distance, which can be reduced to the bulk quasihole tunneling amplitude

result reported earlier. $\frac{6}{6}$ The tunneling amplitude of topological nature is robust against the influence of long-range interaction and disorder. The paper is organized as follows. In Sec. 2, we review our previous study about the bulk quasihole tunneling amplitude in Laughlin state and explain the technical details. The study is extended to edge quasihole tunneling amplitude in Sec. 3. The robustness of the tunneling amplitude in the presence of long-range coulomb interaction and disorder is emphasized in Sec. 4. We summarize the results in Sec. 5 .

\section{A brief review of the bulk quasihole tunneling amplitude in a Laughlin droplet}

We consider a FQH liquid on a disk and assume a single-particle potential $V_{\text {tunnel }}(\theta)=V_{t} \delta(\theta)$, which breaks the rotational symmetry. The potential defines a 
tunneling path for quasiparticles under the gate influence at a quantum point contact. We start by quoting the result $\sqrt{5}$ for the tunneling matrix element between two single-particle states with angular momentum $k$ and $l, v_{p}(k, l) \equiv\left\langle k\left|V_{\text {tunnel }}(\theta)\right| l\right\rangle=$ $\frac{V_{t}}{2 \pi} \frac{\Gamma((k+l) / 2+1)}{\sqrt{k ! l !}}$. The tunneling operator of the many-body wavefunction is then defined as $\mathcal{T}=\sum_{i} V_{\text {tunnel }}\left(\theta_{i}\right)$ and the amplitude for a quasihole to tunnel to the droplet edge is

$$
\Gamma_{q h}=\frac{\left\langle\Psi_{q h}|\mathcal{T}| \Psi_{0}\right\rangle}{\sqrt{\left\langle\Psi_{q h} \mid \Psi_{q h}\right\rangle} \sqrt{\left\langle\Psi_{0} \mid \Psi_{0}\right\rangle}}
$$

where $\left|\Psi_{0}\right\rangle$ and $\left|\Psi_{q h}\right\rangle$ are the wavefunctions for the ground state and the quasihole state, respectively.

The matrix element form of the tunneling amplitude suggests that we can either use Lanczos-type exact diagonalization or variational Monte Carlo simulation to calculate. However, these approximations fail to reach the accuracy needed for the error-free determination of the conformal dimensions of quasiholes (though they are useful in the discussion of the long-range interaction and disorder effects). Fortunately, the application of Jack polynomial provides an instructive yet numerical exact calculation method.

Let us digress and explain first the connection between the Laughlin model wavefunction, which is the exact ground state of the hard-core ( $V_{1}$ only) interaction, and the Jack polynomial ${ }^{7}$ In general, Jacks belong to a family of symmetric multivariate polynomials of the complex particle coordinates. Potentially, they can be FQH wavefunctions for bosons (appending the ubiquitous Gaussian factor) or for fermions (with an extra antisymmetric factor $\prod_{i<j}\left(z_{i}-z_{j}\right)$, i.e., the Vandermonde determinant). A Jack $J_{\lambda}^{\alpha}\left(z_{1}, z_{2}, \cdots, z_{N}\right)$ can be parametrized by a rational number $\alpha$ (negative in this context), which is related to the clustering properties of the polynomial wavefunction, and a root configuration $\lambda$, which satisfies a generalized Pauli exclusion principle and from which one can derive a set of monomials that form a basis for the Jack. The Jack is an eigenstate of the corresponding CalogeroSutherland Hamiltonian

$$
H_{C S}=\sum_{i}\left(z_{i} \partial_{i}\right)^{2}+\frac{1}{\alpha} \sum_{i<j} \frac{z_{i}+z_{j}}{z_{i}-z_{j}}\left(z_{i} \partial_{i}-z_{j} \partial_{j}\right)
$$

where $\partial_{i} \equiv \partial / \partial z_{i}$. Take the bosonic Laughlin state at $\nu=1 / 2$ (which corresponds to the fermionic Laughlin state at $\nu=1 / 3$ ) for a concrete example. One can easily check that $\prod_{i<j}\left(z_{i}-z_{j}\right)^{2}$ satisfies $H_{C S}$ with $\alpha=-2$, which is related to the fact that the bosonic (or the corresponding fermionic) wavefunction vanishes as $\left(z_{i}-z_{i}\right)^{2}$ [or $\left(z_{i}-z_{j}\right)^{3}$ ] when particle $i$ approaches $j$. For two bosons, one obviously has

$$
\left(z_{1}-z_{2}\right)^{2}=z_{1}^{2} z_{2}^{0}-2 z_{1}^{1} z_{2}^{1}+z_{1}^{0} z_{2}^{2}=1 \cdot \mathcal{S}_{2}\left(z_{1}^{2} z_{2}^{0}\right)+(-2) \cdot \mathcal{S}_{2}\left(z_{1}^{1} z_{2}^{1}\right),
$$

which is an expansion of the polynomial wavefunction into a sum of symmetric monomials. The $N$-particle wavefunction $\prod_{i<j}\left(z_{i}-z_{j}\right)^{2}$ can be expanded as

$$
1 \cdot \mathcal{S}_{N}\left(z_{1}^{2 N-2} z_{2}^{2 N-4} \cdots z_{N}^{0}\right)+(-2) \cdot \mathcal{S}_{N}\left(z_{1}^{2 N-3} z_{2}^{2 N-3} z_{3}^{2 N-6} z_{4}^{2 N-8} \cdots z_{N}^{0}\right)+\cdots .
$$


This crude example (perhaps with a more elaborate expansion) illustrates the idea that a bosonic (fermionic) wavefunction can be expanded by a set of homogeneous symmetric monomials (Slater determinants), which can be derived from a single monomial, known as the root, using a squeezing rule, which lowers the relative angular momentum and conserves the total angular momentum for the system with rotational invariance. We choose a numeric string representation for the root configuration, which is simply the collection of occupation numbers of the single-particle orbitals $\left(z^{m}\right.$ in the quantum Hall context). The root configuration for the above example of $N$-particle bosonic Laughlin state is, therefore, $1010 \cdots 101$ and for the corresponding fermioic one $100100 \cdots 1001$. Let us use the convention that the leftmost digit corresponds to the $z^{0}$ orbital, i.e., the droplet center. It is closely related to the topological nature of the wavefunction that the mere knowledge of a Jack parameter and a matching root configuration are enough to generate the coefficients of all the descendant symmetric monomials (Slater determinant) numerically exact - practically to more than 10 particles - with a recursive method 8

The FQH model wavefunctions can also be written as the correlators of certain primary fields in some conformal field theories. For example, the Laughlin wavefunction at filling fraction $\nu=1 / M$ can be constructed by the chiral boson conformal field theory (CFT) with a compactification radius $M ! 9$ The primary fields are vertex operators $e^{i m \varphi(z) / \sqrt{M}}$, where $\varphi(z)$ is a chiral boson field. Operators with $m=1,2, \ldots M-1$ correspond to quasiholes with different charge, whose corresponding conformal dimensions are $\Delta=m^{2} /(2 M)$. It is, therefore, reasonable to expect that the tunneling amplitude $\Gamma$ as a function of system size $N$ (with a given tunneling distance $d$ ) may show scaling behavior, $\Gamma \sim N^{\beta}$, whose scaling exponent $\beta$ is related to the conformal dimension of the tunneling particle. Our previous work 6 confirmed the hidden (due to the dominant single-particle effect) scaling behavior of the quasihole tunneling amplitude, $\beta=1-2 \Delta$, which can be explained by the effective field theory consideration that the tunneling amplitudes contains factors of quasiparticles propagating along the opposite edges.

In the identification of the relation between the scaling exponent and the conformal dimension of the corresponding quasiparticle, the exact calculation of the tunneling amplitude $\Gamma=\left\langle\Psi_{q h}|\mathcal{T}| \Psi_{L}\right\rangle /\left(\sqrt{\left\langle\Psi_{q h} \mid \Psi_{q h}\right\rangle} \sqrt{\left\langle\Psi_{L} \mid \Psi_{L}\right\rangle}\right)$ using the Jack polynomial method plays a crucial role, which, in fact, motivated the effective field theory interpretation. 6 The exactness allowed the elimination of all finite-size uncertainties with the conjecture of an exact tunneling amplitude at the small interedge limit, at which we deform the $N$-particle system (with a puncture, or quasiholes, at the center) into a ribbon, or topologically $S^{1} \times[0, d]\left(d / l_{B} \ll N\right)$. This effectively erases the dominant single-particle effect (i.e. the Gaussian Landau level form factor). In this limit, for $M=3$ or $\nu=1 / 3$, we conjectured 6 that the tunneling amplitude for the charge- $e / 3$ quasihole is

$$
2 \pi \Gamma_{L, M}^{e / M}(N)=\frac{N}{M} B\left(N, \frac{1}{M}\right)
$$


where $M=3$ and $N$ is the number of electrons. Here we introduce the beta function $B(x, \eta)=\Gamma(x) \Gamma(\eta) / \Gamma(x+\eta)$ which, for large $x$ and fixed $\eta$, asymptotically approaches $\Gamma(\eta) x^{-\eta}$, where $\Gamma(x)$ is the Gamma function (not the tunneling amplitude elsewhere). We verified numerically that the conjecture is exact for up to 10 electrons; therefore, asserting the conjecture is also exact for larger system, we obtain the exact exponent $\beta^{(e / 3)}=1-1 / 3=2 / 3$ in the scaling behavior $\Gamma^{(e / 3)} \sim N^{\beta^{(e / 3)}}$. This is also verified to be applicable for $M=5$ a In other words, based on the scaling analysis we discussed earlier, we can compute the conformal dimension of smallest charged quasiholes in the $\nu=1 / M$ Laughlin state to be $\Delta=1 /(2 M)$.

The quasi-one-dimensional ribbon limit essentially removes the unnecessary geometrical information of the wavefunctions, and subsequently reveals a perfect scaling behavior otherwise embedded in inaccuracy and deviations due to small system size. A similar consideration, dubbed as the conformal limit, allows the opening of a full gap in the entanglement spectra of systems on sphere geometry 10 in a way that the low-lying levels showing the universal conformal field theory counting are well separated from the higher Coulomb ones, which are not universal. In both cases, we found that topology stands out after we suppress the geometrical information. In other words, the topological information is encoded in the set of coefficients in Eq. (6), while the geometrical information is encoded in the monomials, which may be deformed, for example, to accommodate the geometry of shear transformation and rotation 11

\section{The edge quasihole tunneling amplitude in a Laughlin droplet}

In the last section, we consider the tunneling amplitude of the bulk quasihole at the center of the droplet. In fact, we can generalize the discussion to quasiholes located elsewhere, or to edge excitations. The generalization is straightforward for the Jack polynomial approach, which applies to low-lying excitations, such as the edge mode in disk geometry 12 and the magnetic-roton mode in sphere geometry! $\frac{13}{13}$ The study of the tunneling properties of edge states $\left(s_{n} \Psi_{L}, n=1,2,3, \ldots\right)$ is an important tool for understanding the topological bulk states both from experimental and theoretical point of view. Here we focus on the tunneling amplitude $\Gamma=\left\langle s_{n} \Psi_{L}|\mathcal{T}| \Psi_{L}\right\rangle$, which crossover smoothly from the tunneling of a bulk quasihole excitation to edge excitations, as $n$ decreases from $N$ to 1 .

The edge mode for the Laughlin state corresponds to a set of states whose root

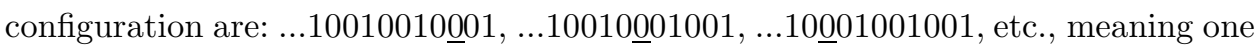
0 (or a quasihole) can be inserted in any one of the 100 unit cells. The tunneling problem we study here is the amplitude for the quasihole in these edge states to tunnel to the outer edge, leaving the Laughlin state behind. Again, we focus on the quasi-one-dimensional ribbon limit to look for a unified analytical solution. As we have already demonstrated the exactness of the method elsewhere,$\frac{6}{6}$ we focus here

${ }^{\text {a}}$ Eq. (7) also applies to the integer case $(M=1)$, in which the righthand side reduces to unity. 
on presenting the analytical conjecture on the tunneling amplitude, which has been verified to be correct for all accessible system sizes.

For the consistency with Eq. (7), we define,

$$
T(N) \equiv T(N, 1 / M)=\sqrt{\frac{2 \pi}{M} \Gamma_{L, M}^{e / M}(N)}=\frac{1}{M} \sqrt{N B\left(N, \frac{1}{M}\right)},
$$

for $N \geq 0$ and specifically define $T(0,1 / M)=1 / M$. This allows us to unify the edge and bulk quasihole tunneling amplitudes as

$$
2 \pi \Gamma_{L, M}^{e / M}(N, \Delta k)=\frac{T(N) T(\Delta k)}{T(N-\Delta k)},
$$

where the integer $\Delta k$ is the angular momentum of the edge/quasihole excitation (i.e., the number of $1 \mathrm{~s}$ to the right of the inserted 0 ). For example, if we consider a system with a root configuration $10010010010 \underline{001001}$ and a Jack parameter $\alpha=-2$, the additional parameters can be read as $N=6, M=3$, and $\Delta k=2$. When $\Delta k=N$, it recovers Eq. (7), as the additional 0 is located at the leftmost position. Note when $\Delta k=0$, the tunneling amplitude measures the average density, which is $1 / M$. In the thermodynamic limit, for edge excitations, i.e., $\Delta k=O(1)$, we find

$$
2 \pi \Gamma_{L, M}^{e / M}(N, \Delta k)=T(\Delta k)+O(1 / N) .
$$

For bulk excitations, i.e., $\Delta k=O(N)$, we find

$$
2 \pi \Gamma_{L, M}^{e / M}(N, \Delta k) \sim N^{1-1 / M} .
$$

These results allow us to compute straightforwardly the tunneling amplitude for a quasihole anywhere inside a Laughlin droplet to the edge.

\section{Robustness of tunneling amplitude in the presence of realistic edge confinement and disorder}

So far we discussed the exact tunneling results using model wavefunctions generated as Jack polynomials. In other words, the Laughlin wavefunction and its edge states (including the single-quasihole state) are the eigenfunction of the Hamiltonian for electrons with hard-core interaction. In a realistic GaAs/GaAlAs heterostructure, the electrons interact with each other via a long-range coulomb repulsion and are confined by a neutralizing background charge confinement from a doping layer at a setback distance $d$. To see whether the tunneling amplitude is robust, we need to verify the validity of Eq. (7) in the presence of Coulomb interaction. Based on a previous study, 14 we fix the setback distance of the background charge at $d=0.5 l_{B}$, at which the system is in the Laughlin phase, i.e., the global ground state has the same quantum number as that of the Laughlin state and a closeto-unity overlap with the latter as well. On the other hand, the quasihole state is produced by a Gaussian impurity potential $H_{W}=W_{g} \sum_{m} \exp \left(-m^{2} / 2 s^{2}\right) c_{m}^{+} c_{m}$ with a width $s=2 l_{B}, \frac{15}{15}$ which models, e.g., the STM tip potential in an experiment. We 


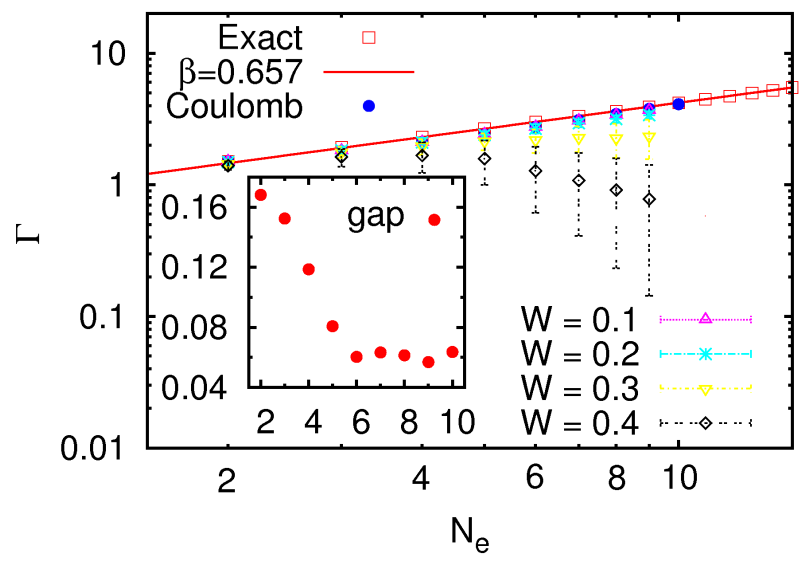

Fig. 1. The tunneling amplitude for the Laughlin phase in the presence of long-range Coulomb interaction and disorder in the quasi-one-dimensional ribbon limit. The exact results for the Laughlin state [Eq. 17] ] can be fit by a power law with an exponent 0.657 (the exact value should be $2 / 3$, which is prone to finite-size error in fitting). In the realistic model with Coulomb interaction and neutralizing charge confinement at a setback distance $d=0.5 l_{B}$ (solid points), we obtain almost the same $\Gamma$ as using the model wavefunctions. When we include the disorder potential with strength $W, \Gamma$ remains unchanged at weak disorder, but deviates from the exact values at strong disorder. The inset plots the energy gap between the ground state and the first excited state in the same angular momentum subspace with $M=3 N(N-1) / 2$ of the pure Coulomb Hamiltonian with $d=0.5 l_{B}$.

calculate the bulk quasihole tunneling amplitude up to 10 electrons and compare it (deformed to the quasi-one-dimensional ribbon limit) with Eq. (7). As shown in Fig. 1, the long-range coulomb interaction has very little effect on the tunneling amplitude, hence the scaling behavior of Eq. (77) is, to a good approximation, valid for the realistic interaction, as long as the system remains in the Laughlin phase.

In addition, a realistic system also contains impurity scatterings. Nevertheless, the topological properties of an FQH state is believed to be robust against weak disorder. To prove the statement regarding disorder, we consider an uncorrelated random potential on each Landau level orbital, such that $H_{D}=\sum_{m} U_{m} c_{m}^{+} c_{m}$, where $U_{m}$ denotes the random potential on the $m$ th orbital, whose value is randomly chosen in the range of $[-W / 2, W / 2]$. We compute the tunneling amplitudes by averaging over more than 1000 random samples for a given disorder strength $W$. As shown in Fig. 1 the tunneling amplitudes for weak disorder, i.e., $W=0.1$, are almost the same as that in the pure coulomb case for all accessible system sizes. However, when we increase the strength of disorder gradually, the tunneling amplitude $\Gamma$ deviates significantly from the exact results for large enough system size and therefore, the scaling hypothesis of Eq. (7) fails in the strong disorder case. To quantitatively understand the disorder effect, we can define and compute the energy gap for the system as the energy difference between the ground state (which 
is the Laughlin-like state) and the first excited state in the same subspace with a total angular momentum $3 N(N-1) / 2$. As shown in the inset of Fig. 1 when we fix $d=0.5 l_{B}$, the energy gap has very little finite size fluctuations for $N>6$; the gap is around $0.06 e^{2} / \epsilon l_{B}$. Therefore, disorder starts to affect the tunneling amplitudes when the strength of disorder is significantly larger than the energy gap (up to an $O(1)$ prefactor). This, in return, suggests that the deviation of the tunneling amplitude from the scaling behavior can be explored to study the transition of the FQH phase to insulator, much like the Chern number study of the FQH-insulator transition! 16

\section{Conclusions}

In summary, we calculate the tunneling amplitude for quasihole in the Laughlin phase, generalizing the previous result for the tunneling of a quasihole at the center of a circular Laughlin droplet to an arbitrary location. This is achieved by considering the tunneling amplitudes between the Laughlin state and its accompanying edge states. Using the exact Jack polynomial expansion, we showed that the bulk and edge quasihole tunneling can be unified by a single equation (9) for any system size. We also demonstrate that the quasihole tunneling amplitude is robust against realistic considerations, such as the long-range coulomb interaction, neutralizing background charge confinement, and moderate amount of disorder.

\section{Acknowledgements}

This work was supported by the National Basic Research Program of China (973 Program) grant No. 2009CB929100, National Natural Science Foundation of China (NSFC) grant No. 11174246, and US DOE grant No. de-sc0002140. KHL acknowledges the support at the Asia Pacific Center for Theoretical Physics from the Max Planck Society and the Korean Ministry of Education, Science and Technology.

\section{References}

1. F. Wilczek, Phys. Rev. Lett. 48, 1144 (1982).

2. B. I. Halperin, Phys. Rev. Lett. 52, 1583 (1984).

3. D. Arovas, J. R. Schrieffer, and F. Wilczek, Phys. Rev. Lett. 53, 722 (1984).

4. A. Kitaev, Ann. Phys. 303, 2 (2003).

5. H. Chen, Z.-X. Hu, K. Yang, E. H. Rezayi, and X. Wan, Phys. Rev. B 80, 235305 (2009).

6. Z.-X. Hu, K.-H. Lee. E. H. Rezayi, X. Wan and K. Yang, New J. Phys. 13, 035020 (2011).

7. B. A. Bernevig and F. D. M. Haldane, Phys. Rev. Lett. 100, 246802 (2008). B. Bernevig and F. Haldane, Phy. Rev. Lett. 101, 246806 (2008). B. A. Bernevig and N. Regnault, Phys. Rev. Lett. 103, 206801 (2009).

8. R. Thomale, B. Estienne, N. Regnault, and B. A. Bernevig, Phys. Rev. B 84, 045127 (2011).

9. G. Moore and N. Read, Nucl. Phys. B 360, 362 (1991). 
10. R. Thomale, A. Sterdyniak, N. Regnault, and B. A. Bernevig, Phys. Rev. Lett. 104, 180502 (2010).

11. N. Read and E. H. Rezayi, Phys. Rev. B 84, 085316 (2011).

12. K.-H. Lee, Z.-X. Hu, and X. Wan, unpublished.

13. B. Yang, Z.-X. Hu, Z. Papić, and F. D. M. Haldane, unpublished.

14. X. Wan, K. Yang, and E. H. Rezayi, Phys. Rev. Lett. 88, 056802 (2002).

15. Z.-X. Hu, X. Wan, and P. Schmitteckert, Phys. Rev. B 77, 075331 (2008).

16. D. N. Sheng, X. Wan, E. H. Rezayi, K. Yang, R. N. Bhatt, and F. D. M. Haldane, Phys. Rev. Lett. 90, 256802 (2003). 